\title{
Influence du procédé d'hydrolyse des protéines de poisson sur leur utilisation par le veau de boucherie. Comparaison avec les protéines de lait et celles de soja
}

\author{
R. TOULLEC et P. PATUREAU-MIRAND*
}

avec la collaboration technique de

\author{
Marguerite Braurils, P. Gaucher, J. LAREYNIF, Odile BFrnard* ${ }_{2}$ et \\ Marie-Claude ValdicY*
}

Station de Recherches Zootechniques

Centre de Recherches de Rennes, I.N.R.A., $6_{5}$, vue de Saint-Brieuc, $35^{\circ} 4^{2}$ Rennes Cedex (France)

* Laboratoive d' Études du Métabolisme Azoté, Centre de Recherches de Clermont-Ferrand, I.N.R.A., Theix, 63i Io Beaumont (France)

\begin{abstract}
Résumé
L'utilisation de 3 concentrats protéiques (Poisson A, Poisson B et Soja) a été étudiée chez le veau de boucherie. Les 2 premiers concentrats ont été préparés à partir de poissons blancs, par hydrolyse enzymatique ménagée, à l'aide d'un mélange d'enzymes protéolytiques végétales (A) ou bactériennes (B). Le concentrat de soja était un tourteau cuit débarrassé de ses oligosaccharides par extraction éthanolique.

Quatre aliments d'allaitement contenant environ 24 p. Ioo de matières azotées et $18 \mathrm{p}$. I oo de matières grasses par rapport à la matière sèche, ont été préparés. Dans l'aliment Témoin, les protéines provenaient presque exclusivement du lait. Dans les 3 autres aliments (Poisson A, Poisson B et Soja), 59 p. roo des protéines étaient apportées par l'un des concentrats protéiques étudiés, le reste étant fourni par la poudre de lait écrémé, la lysine et la méthionine. Chacun de ces aliments a été distribué à I 2 veaux mâles de race frisonne, entre les âges d'environ 3 et 16 semaines.

Les performances des veaux du lot Poisson $\mathrm{A}$ ont été inférieures à celles des animaux du lot Témoin mais seule la différence observée dans l'efficacité alimentaire $(6,5 \mathrm{p}$. I oo pour l'ensemble de l'essai) a été significative. Les résultats concernant les veaux du lot Poisson B ont été moins satisfaisants que ceux obtenus avec les animaux du lot Poisson A $(-8,6 ;-4,9$ et -5 , I p. Ioo pour le gain de poids vif, l'efficacité alimentaire et le poids de carcasse). Les performances des veaux du lot Soja ont été très voisines de celles des animnaux du lot Poisson $B$ et donc inférieures à celles des veaux des lots Témoin et Poisson A. La valeur de l'hématocrite a moins diminué chez les veaux des lots Poisson B et Soja que chez ceux des lots Poisson A et surtout Témoin.

L'effet dépressif observé en remplaçant les protéines du lait par celles des concentrats protéiques étudiés pourrait être dû en partie à un apport protidique insuffisant, au moins au cours des premières semaines d'essai, comme l'indique l'urémie plus faible de ces animaux. Il s'expliquerait également par un moins bon équilibre en acides aminés indispensables et semi-indispensables, surtout dans le cas des concentrats protéiques de poisson, comme le suggère l'examen de
\end{abstract}


1'aminoacidémie des veaux. L'utilisation moins satisfaisante du concentrat de poisson B, par rapport au concentrat $A$, pourrait être due à une moindre disponibilité de la valine.

Les concentrats protéiques préparés par hydrolyse enzymatique à partir de poissons blancs, peuvent remplacer une part importante du lait écrémé dans les aliments d'allaitement destinés au veau de boucherie. Cependant, pour éviter tout effet dépressif, il conviendrait de ne pas dépasser un taux de substitution voisin de $35 \mathrm{p}$. Ioo, après avoir associé les concentrats à la poudre de lactosérum, afin d'obtenir un mélange mieux équilibré en acides aminés indispensables. Le type d'enzymes employées pour l'hydrolyse a un effet important sur l'utilisation des protéines. Le concentrat de soja étudié dans cet essai n'est pas aussi bien utilisé que le concentrat de poisson A.

\section{Introduction}

Les concentrats protéiques obtenus à partir des poissons blancs par hydrolyse enzymatique ménagée pourraient remplacer une part importante de la poudre de lait écrémé dans les aliments d'allaitement destinés au veau préruminant. Ils sont en majeure partie solubles, propriété intéressante pour leur introduction dans des aliments distribués sous forme liquide. Leur digestibilité apparente est élevée (9I p. Ioo pour l'azote à partir de 1'âge d'un mois, d'après PARUELIE et al., I974; Touliec et al., i977; Toullec, Corolifer et Patureau-Mirand, i977). Ils permettent d'obtenir des performances satisfaisantes lorsqu'ils apportent environ la moitié des protéines alimentaires, en association avec la poudre de lactosérum (PARUELLE et al.; I974; TroccoN et Toul, EC, I976), malgré leur teneur peu élevée en acides aminés indispensables et semi-indispensables (PATUREAUMIRAND et al., I974).

Le tourteau de soja est une source abondante de protéines relativement mieux équilibrées en acides aminés (PION et FAUCONNEAU, I 966) mais moins digestibles que celles du poisson (74 p. IOO d'après PARUFLLE et al., I972), même lorsque le saccharose et les $\alpha$ galactosides ont été éliminés par extraction à 1'alcool (80 p. Ioo, d'après GUILLOTEAU et al., I977).

Dans cet essai, nous avons étudié l'utilisation par le veau de boucherie, de 2 concentrats protéiques de poissons blancs obtenus à l'aide de 2 préparations enzymatiques différentes, en comparaison avec la poudre de lait écrémé et un concentrat protéique de soja.

\section{Matériel et méthodes}

\section{Aliments}

Les 2 concentrats protéiques de poisson utilisés ont été préparés à partir de déchets de filetage de poissons blancs par hydrolyse enzymatique ménagée, suivie d'abord de 2 centrifugations pour éliminer partiellement les protéines demeurées insolubles et les lipides, puis d'un séchage par le procédé Spray. L'un d'entre eux (A) était un produit commercial préparé à l'aide d'un mélange d'enzymes protéolytiques végétales. L'autre (B) provenait d'une fabrication d'essai réalisée 
dans les mêmes installations en utilisant des enzymes protéolytiques bactériennes. Les produits $\mathrm{A}$ et $\mathrm{B}$ avaient des teneurs voisines en matières azotées, en phosphore, en calcium, en magnésium et en fer (tabl. I); en revanche, le second était moins pourvu en lipides et plus riche en sodium, en potassium et en chlorures.

Le concentrat protéique de soja était un produit commercial obtenu en extrayant le saccharose et les $\alpha$ galactosides du tourteau par l'éthanol aqueux à chaud et en appliquant un traitement thermique pour éliminer les facteurs antinutritionnels; 1'échantillon utilisé contenait 63,9 p. Ioo de matières azotées par rapport à la matière sèche (tabl. I).

\section{TABLEAU I}

Composition des concentrats protéiques ( $p$. roo de la matière sèche)

Composition of protein concentrates (per cent of dry matter)

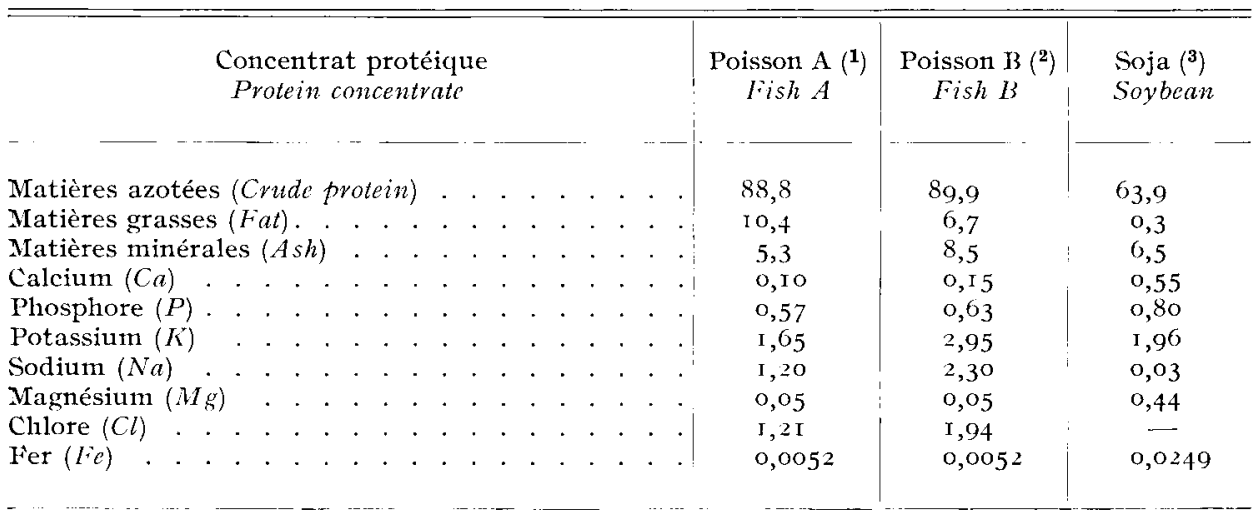

( 1 et 2) Concentrats solubles de poisson (Soluble Fish concentrates) Coopérative de Traitement des Produits de la Pêche, BP 36I/2, 62203 Boulogne-sur-Mer.

(3) Haypro-T, Hayes I,T D, Ashood, Israel.

I a composition en acides aminés des concentrats protéiques a été déterminée selon la méthode décrite par Pion et FAuconxeau (I966); le tryptophane a été dosé à 1'aide d'une technique dérivée de celle de SLump et SchrEUdER (Ig69). La somme des teneurs corrigées en acides aminés indispensables et semi-indispensables est peu élevée dans les matières azotées des concentrats de poisson, particulièrement dans le produit $\mathrm{B}$ (tab1. 2). Les matières azotées du concentrat de soja sont mieux pourvues en acides aminés indispensables, sauf en acides aminés soufrés et en lysine.

Quatre aliments d'allaitement contenant environ $24 \mathrm{p}$. I oo de matières azotées et $\mathrm{I} 8 \mathrm{p}$. Ioo de matières grasses ont été préparés (tab1. 3). Dans l'aliment Témoin, les protéines provenaient presque exclusivement du lait écrémé. Dans les 3 autres aliments (Poisson A, Poisson B et Soja), environ 59 p. Ioo des protéines étaient apportées par l'un des concentrats protéiques étudiés, le reste étant fourni principalement par la poudre de lait écrémé. Les 4 aliments ont été supplémentés en méthionine et les aliments Poisson et Soja en lysine, compte tenu des besoins du veau proposés par Patureau-Mirand et al. (I974). Les aliments Poisson A et Poisson B étaient moins riches que l'aliment 'Témoin en acides aminés indispensables et semi-indispensables (tabl. 2); seules, les teneurs en acides aminés soufrés et 


\section{TABLEAU 2}

Composition on acides aminés des concentrats protéiques et des aliments Amino-acid composition of protein concentrates and of diets $(g / 16 g N)$

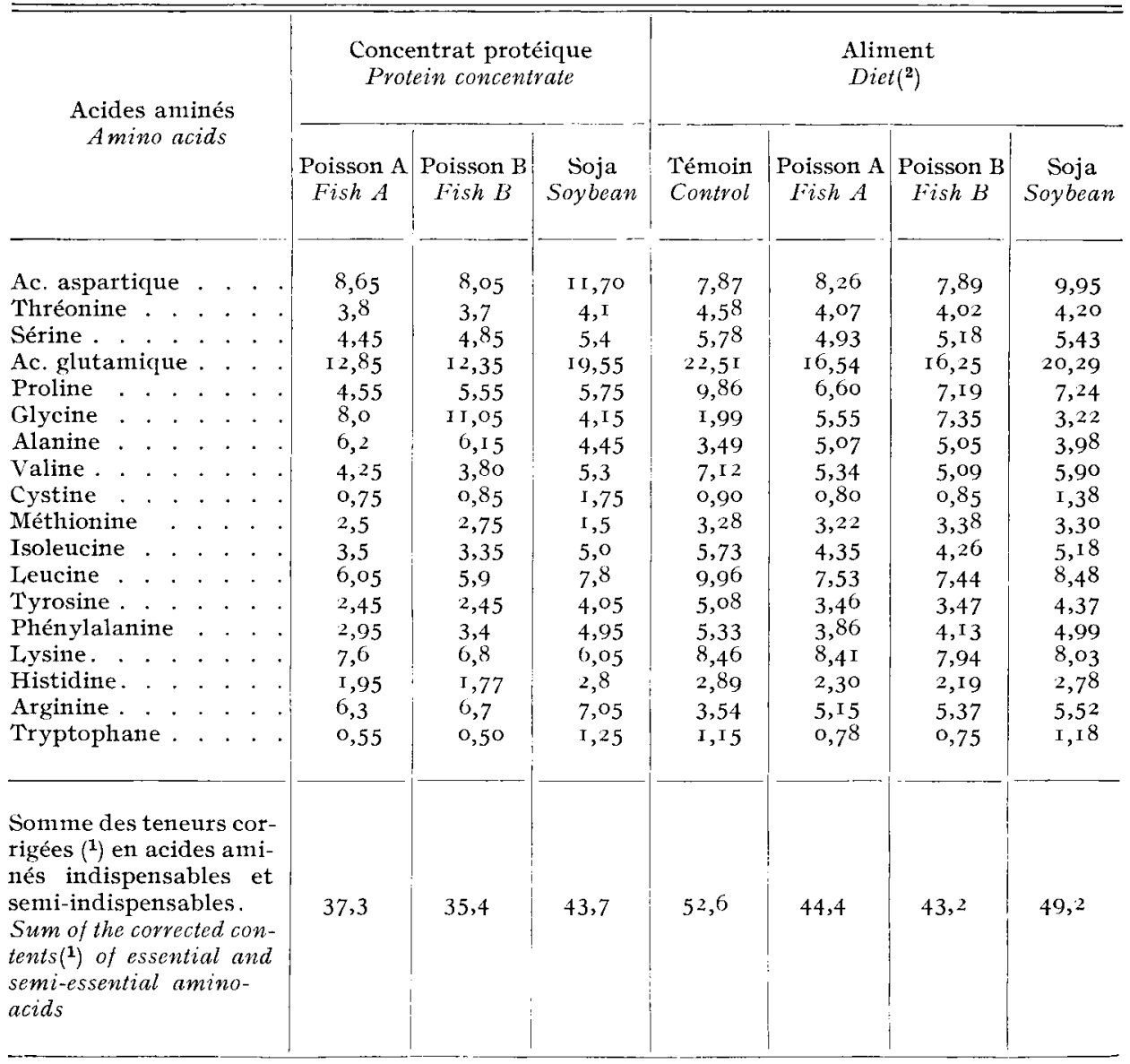

(r) Teneur en leucine limitée à 1,5 fois celle en isoleucine; somme des teneurs en tyrosine et phénylalanine limitée à 12 p. roo de la somme des acides aminés indispensables et semi-indispensables; teneur en arginine limitée à 7,3 p. roo de la somme des teneurs en acides aminés indispensables et semi-indispensables (PION, DE BELSUNCE et FAUCONNEAU, 1963) (Leucine content limited to 1.5 of that of isoleucine; sum of tyrosine and phenylalanine contents limited to $I 2 \mathrm{p}$. Ioo of the sum of essential and semi-essential amino-acids. arginine content limited to $7.3 \mathrm{p}$. Ioo? of the sum of essential and semi-essential amino-acids).

(2) Composition calculée à partir de l'analyse des concentrats protéiques et de la composition des protéines du lait (PION et FAUCONNEAU, I 966) (Composition calculated from the analysis of protein concentrates and the composition of milk protein).

en lysine étaient aussi élevées et celle en arginine plus forte dans les aliments Poisson. L'aliment Soja était un peu moins pourvu que l'aliment Témoin en acides aminés indispensables, sauf en acides aminés soufrés, en lysine, en histidine, en arginine et en trytophane; en revanche, il était plus riche que les aliments Poisson en tous les acides aminés indispensables et semi-indispensables, lysine et méthionine exceptées. 
TABLEAU 3

Composition des aliments

Composition of diets

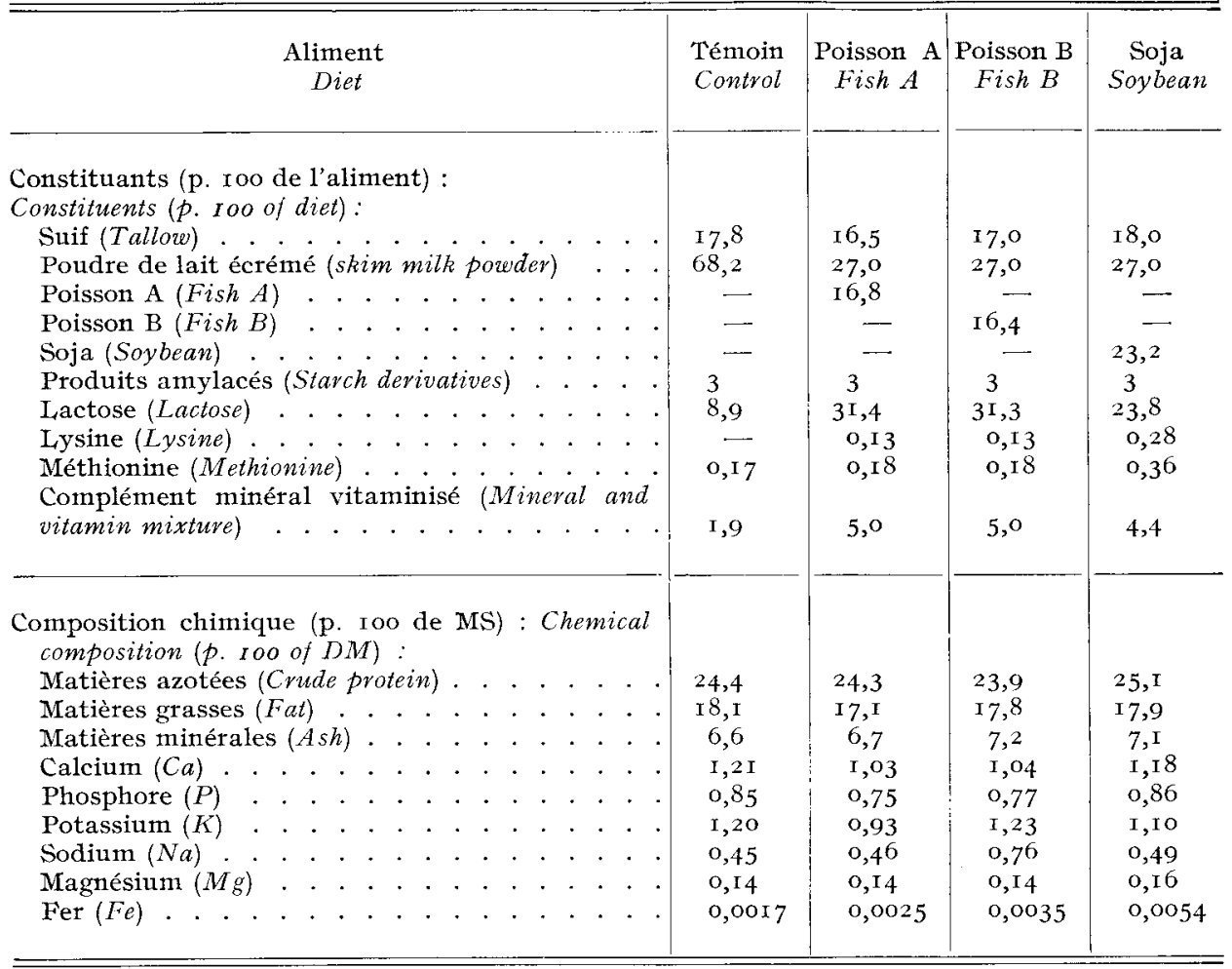

\section{Animaux et Régimes}

48 veaux mâles de race Frisonne ont été achetés à l'âge d'environ 8 jours. Dès leur arrivée, ils ont été munis d'une muselière et placés en cases individuelles sur litière de paille. Après une période d'observation de I2 jours, ils ont été répartis en 4 lots de I2 animaux, compte tenu de leur poids vif, de leur gain de poids vif depuis l'arrivée, de la valeur de leur hématocrite et de leur état sanitaire. Pendant la période d'observation, les veaux ont tous reçu un même lait de remplacement, riche en poudre de lait écrémé, auquel a été progressivement substitué l'un des 4 aliments expérimentaux à partir du quinzième jusqu'au dix-neuvième jour. Les animaux ont été alimentés selon leur poids vif et leur appétit. La concentration est passée progressivement de I30 à I $90 \mathrm{~g}$ d'aliment $/ \mathrm{kg}$ de lait de remplacement et la quantité de matière sèche proposée de 860 à $2920 \mathrm{~g} / \mathrm{j}$. Les aliments ont été distribués au seau à raison de 2 repas par jour; le repas du dimanche soir a été supprimé à partir de la quatrième semaine de présence en étable. 


\section{Prélivements et Mesures}

Les animaux ont été pesés dès leur arrivée, le douzième jour, puis une fois par semaine à heure fixe et avant l'abattage. Quatre prélèvements de sang ont été effectués dans la veine jugulaire une semaine avant et cinq, neuf et douze semaines après la mise en lots. Ils ont eu lieu entre 4 h et $4 \mathrm{~h} 30$ après le repas du matin, sauf le premier qui a été réalisé I h 30 plus tard. La valeur de l'hématocrite a été déterminée sur tous les échantillons et les taux plasmatiques de glucose et d'urée sur les échantillons des premier et second prélèvements (MICHEL, I973). Les teneurs en acides aminés libres ont été mesurées lors des 3 derniers prélèvements, sur un échantillon moyen par lot, préparé en mélangeant les sangs des 8 veaux ayant eu la croissance la plus rapide pendant la semaine précédente (PATUREAUMiRand et al., I974).

I’abattage des veaux a été effectué 93 et 94 jours après la mise en lots, à raison de la moitié de l'effectif de chaque lot par journée. La carcasse chaude, le foie et les dépôts adipeux périrénaux ont été pesés. $\mathrm{I}_{\text {a }}$ longueur de la carcasse a été mesurée et la coloration de la viande a été notée subjectivement.

\section{Résultats}

\section{Etat sanitaire et appétit}

Pour l'ensemble de la période expérimentale, l'état sanitaire des animaux a été très satisfaisant et les refus alimentaires ont été peu importants (respectivement $\mathrm{I}, 4-0,9-\mathrm{I}, 6$ et 0,6 p. Ioo des quantités proposées chez les animaux des lots Témoin, Poisson A, Poisson B et Soja).

\section{Croissance et efficacité alimentaire (tabl. 4)}

Les performances des veaux du lot Poisson A ont toujours été inférieures à celles des animaux du lot 'Témoin mais seules les différences observées dans l'efficacité alimentaire ont été significatives (au cours des 4 premières semaines et pour l'ensemble de l'essai). De même, les résultats des animaux du lot Poisson B ont été moins satisfaisants que ceux des veaux du lot Poisson $A$, surtout au cours des 8 premières semaines; pour l'ensemble de l'essai, le gain de poids vif a été inférieur de $8,6 \mathrm{p}$. Ioo et l'efficacité alimentaire de 4,9 p. Ioo. Les performances des animaux du lot Soja ont été très voisines de celles des veaux du lot Poisson $B$ et donc inférieures à celles des veaux des lots Témoin et Poisson A.

\section{Valeur de l'hématocrite, urémie et glycémie (fig. I)}

La valeur de l'hématocrite a diminué pendant les io premières semaines de l'essai, surtout chez les veaux des lots Témoin et Poisson A. La valeur observée avant 1'abattage a été significativement plus élevée chez les veaux des lots Poisson B et Soja que chez ceux des lots Témoin et Poisson A.

Les taux plasmatiques d'urée et de glucose ont été significativement moins 


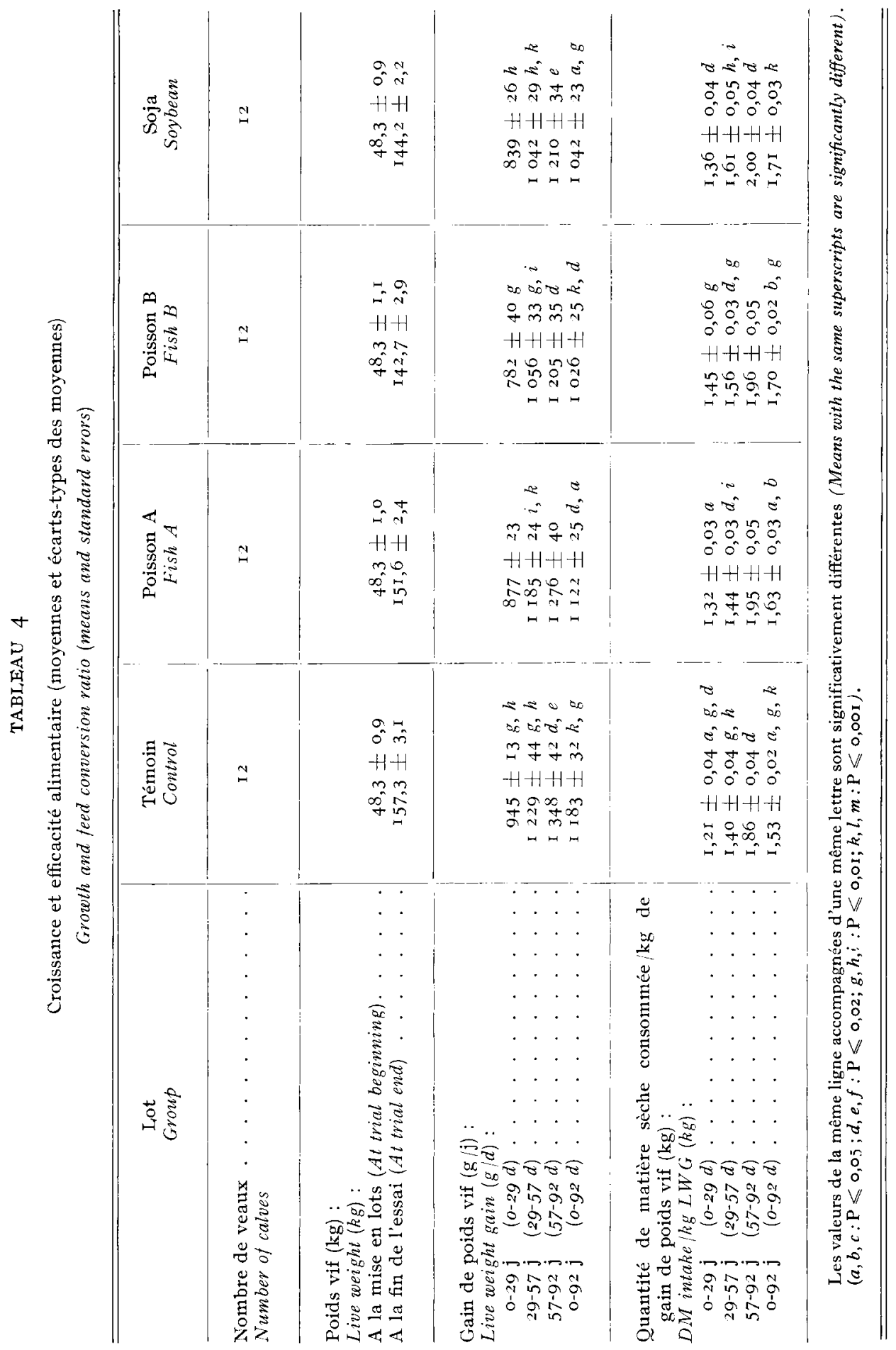


élevés chez les veaux des lots Poisson et Soja que chez ceux du lot 'Témoin. Aucune différence n'a été observée entre les animaux des lots Poisson A, Poisson B et Soja.

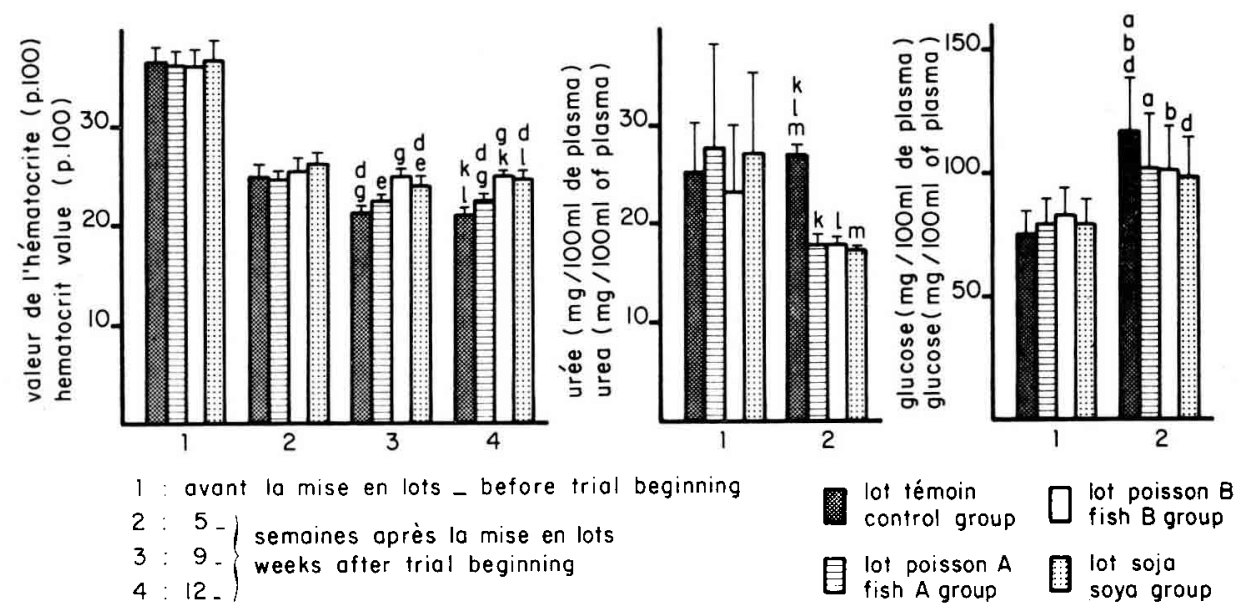

FIG. I. - Évolution de la valeur de l'hématocrite, de l'urémie, et de la glycémie (moyennes é écartstypes des moyennes).

Variation in hematocrit value and in plasma levels of urea and glucose (means and standard errors). Les colonnes surmontées de la même lettre sont significativement différentes (Columns writh the same superscripts are significantly different $)(a, b: \mathrm{P}<0,05 ; d, e: \mathrm{P}<0,02-g, h: \mathrm{P}<0,0 \mathrm{I}$; k. $l . m: \mathrm{P}<\mathrm{o}, \mathrm{OOI}$ ).

\section{Aminoacidémie (tab1. 5)}

Les teneurs en acides aminés libres du sang ont augmenté avec 1'âge, surtout chez les veaux des lots Poisson A, Poisson B et Soja. Cette augmentation a porté presque uniquement sur les acides aminés indispensables et semi-indispensables dans le cas des animaux du lot témoin; en revanche, elle a concerné également les acides aminés non indispensables chez les animaux des 3 autres lots. La somme des teneurs en acides aminés indispensables et semi-indispensables et surtout celle des teneurs en acides aminés non indispensables ont toujours été plus élevées dans le sang des veaux des 2 lots Poisson que dans celui des animaux du lot Témoin. Dans le cas du lot Soja, ces sommes ont été plus faibles que dans celui du lot Témoin pendant la cinquième semaine et un peu plus élevées par la suite; par rapport aux 2 lots Poisson, elles ont toujours été moins élevées, à l'exception de la douzième semaine où la somme des teneurs en acides aminés indispensables a été du même ordre chez les animaux des 3 lots. Aucune différence notable n'a été observée entre les animaux des 2 lots Poisson dans l'évolution avec l'âge de la somme des acides aminés indispensables et de celle des acides aminés non indispensables.

Chez les veaux du lot Témoin, l'augmentation des concentrations sanguines en acides aminés indispensables et semi-indispensables avec l'âge n'a pas porté sur tous les acides aminés : les teneurs en lysine et en histidine n'ont pratiquement pas varié et celles en thréonine et arginine ont diminué. Chez les veaux des 3 autres lots, tous les acides aminés indispensables et semi-indispensables ont augmenté avec 1'âge, à l'exception de la phénylalanine chez les veaux du lot Poisson B. Par rap- 


\begin{tabular}{|c|c|c|c|c|c|c|}
\hline & & $\mathcal{H}$ & 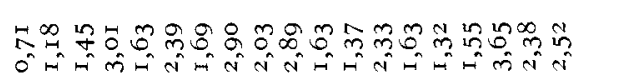 & 8 & $\stackrel{\stackrel{2}{2}}{\hat{2}}$ & $\begin{array}{l}i \\
i 1 \\
\infty \\
\infty \\
\infty\end{array}$ \\
\hline & $\frac{\pi}{\stackrel{5}{*}}$ & $a$ & 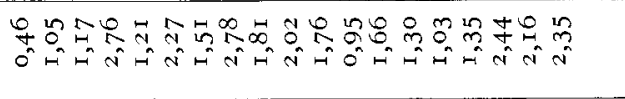 & $\underset{N}{N}$ & $\stackrel{2}{3}$ & $\begin{array}{l}+ \\
\hat{N} \\
\hat{n}\end{array}$ \\
\hline & & in & 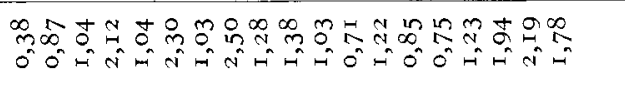 & 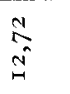 & 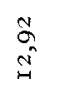 & 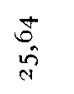 \\
\hline & & $\underset{H}{N}$ & 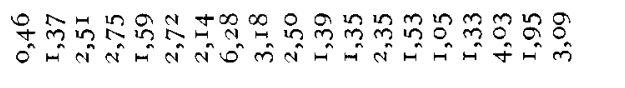 & $\begin{array}{l}\vec{b} \\
\hat{o}^{n} \\
i\end{array}$ & $\begin{array}{l}\mathscr{L} \\
\hat{N} \\
\end{array}$ & in \\
\hline & 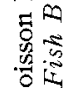 & $a$ & 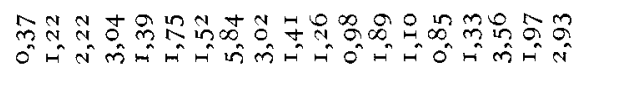 & $\begin{array}{l}\hat{2} \\
\hat{N}\end{array}$ & 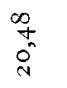 & $\begin{array}{l}\text { b } \\
\hat{m} \\
\hat{m}\end{array}$ \\
\hline & & in & 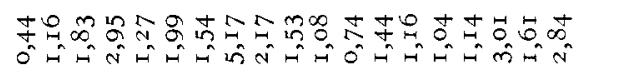 & $\begin{array}{l}\vec{b} \\
\text { in } \\
n\end{array}$ & $\stackrel{\circ}{i n}_{\substack{n \\
\infty}}^{\infty}$ & $\stackrel{H}{\stackrel{f}{m}}$ \\
\hline 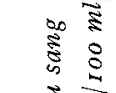 & & $\stackrel{\mathrm{N}}{\mathrm{H}}$ & 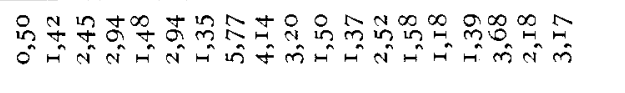 & $D_{\infty}^{\infty}$ & $\begin{array}{l}8 \\
\text { N } \\
\text { N }\end{array}$ & $\begin{array}{l}\stackrel{R}{f} \\
\dot{f}\end{array}$ \\
\hline 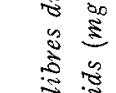 & 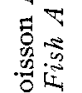 & $\sigma$ & 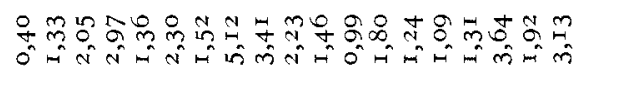 & 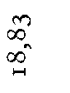 & 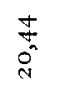 & $\hat{\hat{n}}$ \\
\hline , & & in & 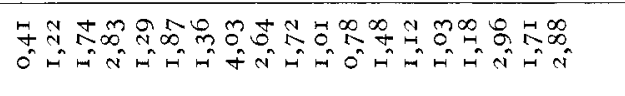 & 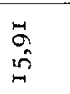 & 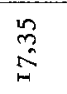 & $\begin{array}{l}\stackrel{0}{i n} \\
\dot{m} \\
m\end{array}$ \\
\hline 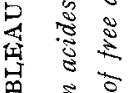 & & $\tilde{H}$ & 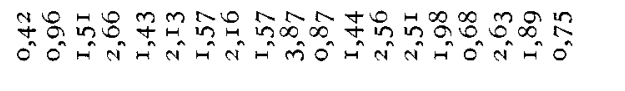 & 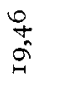 & $\stackrel{m}{\stackrel{m}{F}}$ & $\begin{array}{l}n \\
n \\
\hat{m} \\
m\end{array}$ \\
\hline 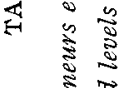 & है & 0 & 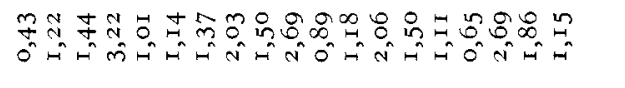 & $\stackrel{n}{2}$ & $\begin{array}{l}2 \\
\hat{s}^{2}\end{array}$ & $\begin{array}{l} \pm \\
\text { in }\end{array}$ \\
\hline 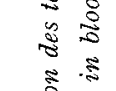 & & in & 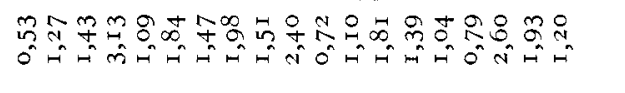 & $\begin{array}{l}0 \\
+ \\
\hat{n} \\
\hat{n}\end{array}$ & $\stackrel{\hat{N}}{\hat{n}}$ & 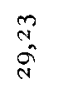 \\
\hline 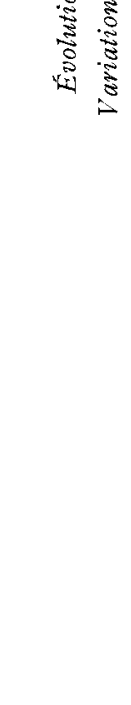 & 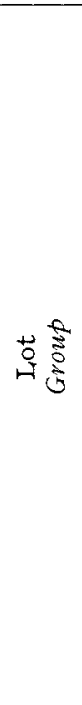 & 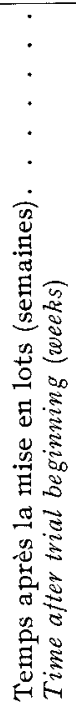 & 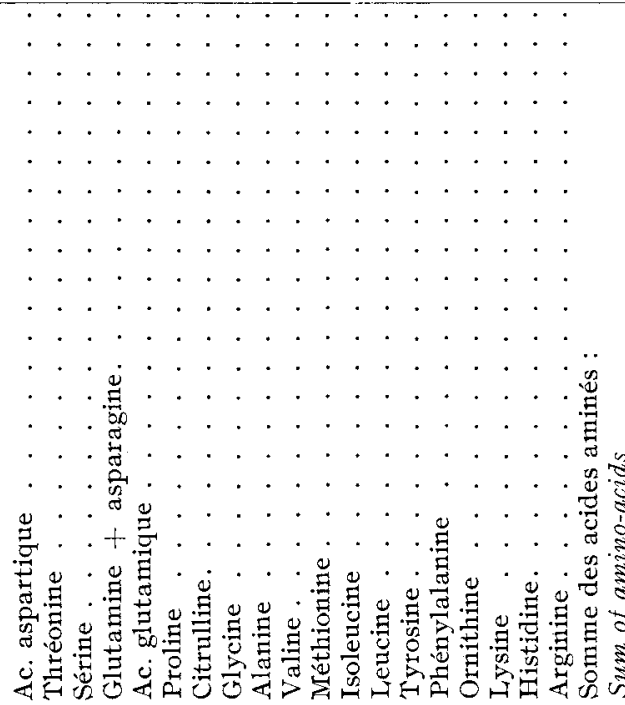 & 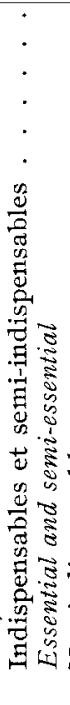 & 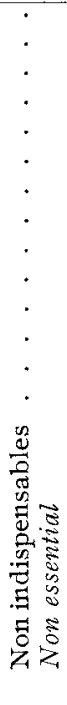 & 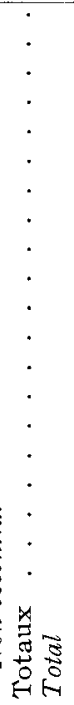 \\
\hline
\end{tabular}


R. TOULLEC, P. PATUREAU-MIRAND

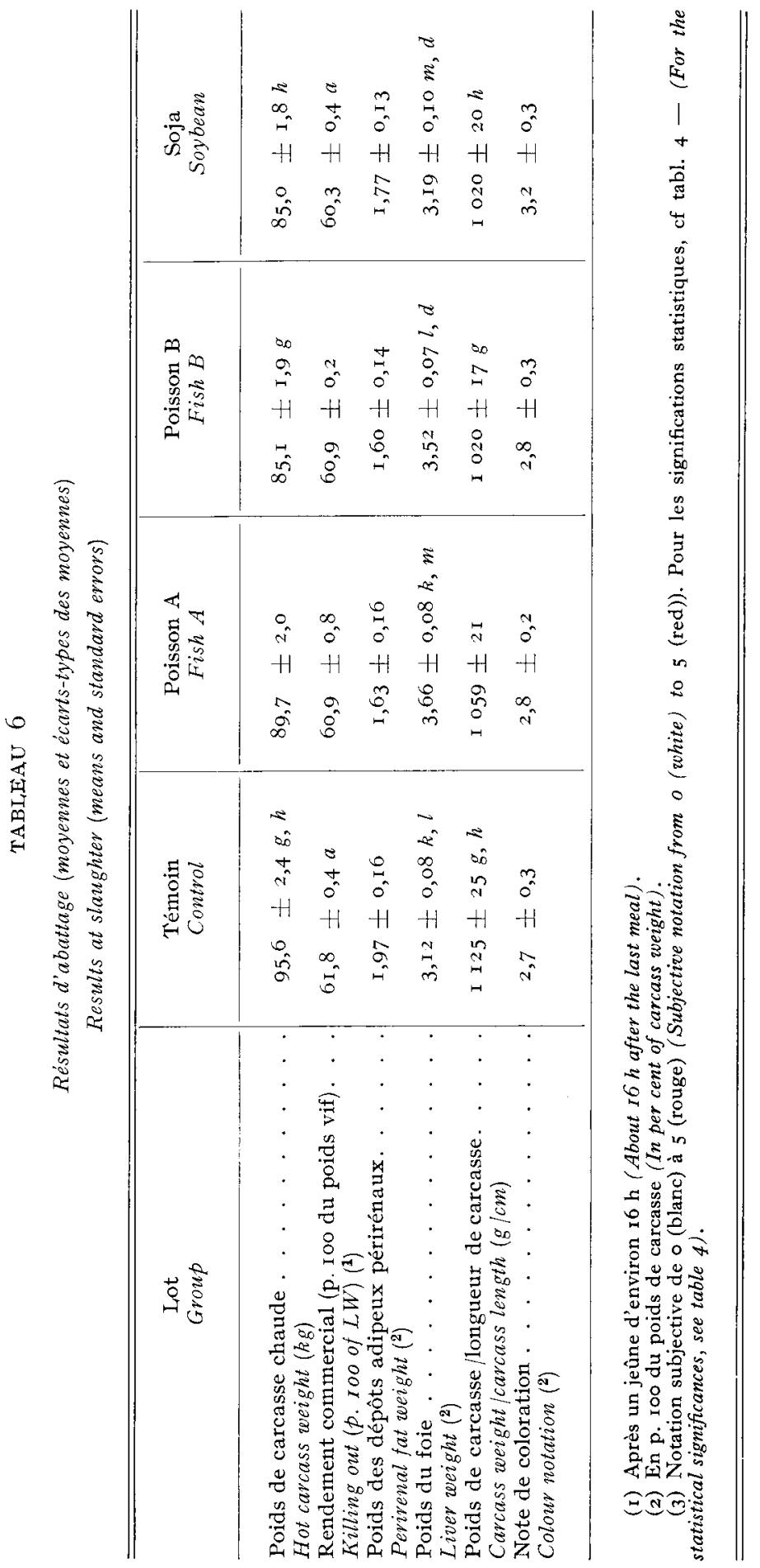


port aux veaux du lot Témoin, ceux des lots Poisson ont toujours eu des teneurs sanguines inférieures en valine, isoleucine, leucine et tyrosine mais supérieures en lysine, méthionine et arginine; il en a été de même chez les veaux du lot Soja, sauf pour la lysine dont le taux sanguin a été inférieur en cinquième et neuvième semaines et supérieur ensuite. Parmi les acides aminés non indispensables, ce sont surtout la glycine et l'ornithine qui ont présenté des teneurs plus importantes dans le sang des animaux recevant les protéines de substitution. Dans le sang des veaux du lot Poisson A, les teneurs en valine et alanine ont été plus fortes et celles en glycine et citrulline plus faibles que chez les animaux du lot Poisson B. Le sang des veaux du lot Soja a toujours été moins pourvu que celui des animaux des lots Poisson en thréonine, sérine, glycine, leucine, lysine et arginine; il a été plus riche en histidine.

\section{Résultats d'abattage (tabl. 6)}

Les animaux du lot Poisson $\mathrm{A}$ ont eu un rendement en carcasse moins élevé, une carcasse et des dépôts adipeux périrénaux plus légers, une conformation appréciée d'après le rapport poids de carcasse/longueur moins satisfaisante, et un foie plus lourd que les veaux du lot Témoin; cependant, seul l'écart observé dans le poids du foie a été significatif. Les animaux du lot Poisson B se sont distingués principalement de ceux du lot Poisson A par un poids de carcasse plus faible mais la différence (5,I p. I0o) n'a pas été significative. En revanche, les écarts entre les animaux du lot Poisson $B$ et ceux du lot 'Témoin ont été significatifs pour le poids de carcasse et celui du foie, ainsi que pour la conformation. Les animaux du lot Soja ont fourni des résultats très voisins de ceux obtenus avec les veaux du lot Poisson B, sauf en ce qui concerne le poids du foie qui a été significativement plus faible. Par rapport aux veaux du lot Témoin, ceux du lot Soja ont eu un rendement en carcasse, un poids de carcasse, une conformation et une coloration moins satisfaisants; seule, la différence observée dans la coloration n'a pas été significative.

\section{Discussion}

Le remplacement de $59 \mathrm{p}$. Ioo des protéines du lait par celles du poisson et du soja a un effet dépressif sur la croissance et l'efficacité alimentaire. Les urémies plus faibles des veaux recevant ces protéines de substitution s'accordent avec les résultats de PATUREAU-MIRAND et al. (I974) et pourraient indiquer un apport protidique insuffisant, du moins au cours des premières semaines de l'essai. En effet, les protéines du poisson et surtout celles du soja sont moins digestibles que celles du lait (PARUELLe et al., I974; Guilioteau et al., I977). La proportion de 1'azote correspondant aux acides aminés, à l'exception de l'azote amidé de la glutamine et de l'asparagine, est plus faible dans les aliments Poisson A et B que dans les aliments Soja et Témoin (respectivement $8 \mathrm{I}, 9-83,8-87, \mathrm{o}$ et 86,7 p. Ioo). En conséquence, les aliments Poisson A, Poisson B et Soja contiennent environ 6 , I - 6,4 et 6,2 p. Ioo d'azote digestible sous forme d'acides aminés de moins que l'aliment Témoin, en admettant que les 2 concentrats de poisson ont la même digestibilité; or, c'est principalement 1'azote des acides aminés qui, au cours de leur catabolisme, participe à la synthèse de 1'urée. 
La glycémie moins élevée des veaux des lots Poisson et Soja par rapport à celle des animaux du lot T'émoin est peut être liée à une stimulation de la sécrétion d'insuline plus importante chez les animaux de ces 3 lots qui ingèrent des protéines dont l'évacuation gastrique (GUILLOTEAU et al., I975 et I979) et la digestion (Patureau-Mrrand, Prugnaud et Pion, i97 i Toullec, Guilloteau et CorolI.ER, I979) sont plus rapides que celles des protéines du lait. Il en résulterait, dans les premières heures qui suivent le repas, un afflux plus important d'acides aminés dans le sang, ce qui favoriserait la sécrétion d'insuline (FAICHNEY et WESTON, I97I).

L'évolution de la valeur de l'hématocrite des veaux recevant les aliments Témoin, Poisson A et Poisson B est normale, compte tenu de la teneur en fer des aliments (tabl. 3). Le fait que la viande des veaux du lot Poisson B ne paraisse pas plus colorée que celle des animaux du lot Témoin est donc surprenant; cela est sans doute dû̀ à l'imprécision de la méthode subjective employée. Bien que l'aliment Soja soit plus riche en fer que l'aliment Poisson B, les valeurs de l'hématocrite sont identiques avec ces 2 régimes; il semble que le fer du concentré de soja soit moins disponible que celui du concentré de poisson $B$, ce qui rejoint les observations de van WEERDEN, HUISMAN et SPRIETSMa (I973). L'augmentation du poids du foie avec l'ingestion des 2 aliments Poisson est difficile à expliquer; elle a déjà été observée avec des aliments contenant des levures d'alcanes (Paruelle, Toullec et Patureau-Mirand, I975). La conformation moins satisfaisante des veaux des lots Poisson et Soja indique que la croissance de leur squelette est moins affectée que celle de leurs muscles.

L'augmentation avec l'âge des teneurs sanguines en acides aminés indispensables et semi-indispensables dans le cas des veaux du lot Témoin ainsi que celle des teneurs en la plupart des acides aminés chez les veaux des autres lots, indiquent que l'utilisation métabolique de ces composés est limitée en fin d'engraissement, probablement par un excès de protéines par rapport à l'énergie. Eun effet, un taux protéique de 24-25 p. Ioo est légèrement excessif en fin d'engraissement (VAN WEERDEN, VAN ES et VAN HELLEMOND, I970).

Aucun des 2 aliments contenant les protéines de poisson n'a d'effet défavorable sur l'appétit des animaux, contrairement aux observations effectuées par WILSON (I972), avec des hydrolysats de poisson préparés à l'aide de papaïne. Les performances des veattx recevant l'aliment Poisson A demeurent relativement satisfaisantes, ce qui s'accorde avec les résultats obtenus par TRoccon et ToviLIC (I976) et par DoDsworTh et al. (I977), en distribuant à des veaux d'élevage des laits de remplacement dans lesquels environ le tiers des protéines étaient apportées par des concentrats protéiques de poisson hydrolysés. L'effet dépressif que nous obtenons est cependant plus important que celui observé chez le veau de boucherie par PARUELLE et al. (I974), en remplaçant la moitié des protéines du lait par un mélange fourni par le même concentrat protéique de poisson (68 p. Ioo) et par la poudre de lactosérum ( $32 \mathrm{p}$. Ioo). Cela pourrait être dî̀ à la moindre richesse des protéines de ce concentrat en la plupart des acides aminés indispensables et semiindispensables, par rapport aux protéines du lait et du lactosérum (PATUREAUMirand et al., I974).

Les concentrations sanguines en méthionine et lysine libres plus élevées chez les veaux du lot Poisson A que chez ceux du lot Témoin, pour des quantités ingérées identiques indiquent que l'utilisation métabolique de ces acides aminés est affectée par un déficit de 1'aliment Poisson A en un ou plusieurs acides aminés indispensables. Ceux-ci sont probablement le tryptophane ou les acides aminés ramifiés ou aromatiques. En effet, le taux de tryptophane est faible dans les protéines $d u$ concentrat $A$; cet acide aminé ne constitue qu'environ $I, 8$ p. roo de la 
somme des teneurs corrigées en acides aminés indispensables et semi-indispensables de l'aliment correspondant, au lieut de 2,2 p. Ioo dans l'aliment Témoin. Les besoins du veau en tryptophane ne sont pas connus mais la supplémentation en cet acide aminé des hydrolysats de poisson blanc permet d'améliorer les performances du porcelet (SEVE et al., I978), dont les besoins en la plupart des acides aminés ne semblent pas très différents de ceux du veau (PATUREAU-Mirand et al., I974). $\mathrm{Au}$ cours de la cinquième et de la neuvième semaines, chez les veaux du lot Poisson A, les concentrations sanguines en valine, leucine et isoleucine sont moins élevées et le rapport de la concentration en tyrosine à celle en phénylalanine est inférieur à $I, 3$, ce qui témoigne généralement d'un apport insuffisant d'acides aminés aromatiques (PATUREAU-MIRAND et al., I974). L'interprétation métabolique des différences d'amino-acidémie selon le régime est cependant délicate, compte tenu de l'évacuation gastrique plus rapide des protéines du soja et surtout de celles du poisson, par rapport à celles du lait (GuILIOTEAU el al., I975 et I979).

Le concentrat protéique de poisson B est un peu moins pourvu en la plupart des acides aminés indispensables et semi-indispensables que le concentrat $A$, mais les différences qui en résultent entre les aliments semblent trop faibles pour expliquer les performances moins satisfaisantes des animaux du lot Poisson B. Les concentrations sanguines en valine et en alanine libres sont nettement plus faibles chez ces animaux, bien que les quantités ingérées ne soient inférieures que de 6 et 2 p. Ioo respectivement. Ces acides aminés dont le premier est indispensable, pourraient donc être moins disponibles dans le concentrat $B$, pour des raisons d'ordre digestif ou métabolique. L'utilisation digestive des protéines du Poisson $\mathrm{B}$ n'a pas été mesurée et n'est peut-être pas aussi élevée que celle des protéines du Poisson $\mathrm{A}$, particulièrement pour certains acides aminés. Il se pourrait que l'emploi des enzymes bactériennes entraîne la formation de composés qui modifient 1'utilisation digestive ou métabolique de certains acides aminés. Il est également possible que l'aliment Poisson B soit trop riche en chlorures (tabl. I).

Les résultats défavorables que nous avons eus en substituant les protéines du soja à celles du lait, sont en accord avec ceux obtenus par MorRILL et al. (I97I) et par NiTSAN et al. (I97I), en utilisant des aliments dans lesquels respectivement 44 et $80 \mathrm{p}$. Ioo des protéines étaient apportées par un concentrat de soja. En revanche, GORRILL et Nicholson (I969) n'ont pas observé d'effet dépressif en remplaçant 7o p. Ioo des protéines du lait par celles d'un concentrat de soja. Ces variations suivant les auteurs peuvent êtres dues aux différences dans le niveau d'alimentation et dans la technologie de préparation des concentrats. L'utilisation moins satisfaisante de l'aliment Soja par rapport à l'aliment Témoin peut s'expliquer par ses teneurs moins élevées en énergie digestible (environ 7 p. roo), ainsi qu'en la plupart des acides aminés indispensables et semi-indispensables, arginine, tryptophane et acides aminés soufrés exceptés (tabl. 2).

Les performances des veaux du lot Poisson A sont plus satisfaisantes que celles des animaux du lot Soja, bien que l'aliment reçu par ces derniers apporte davantage de tous les acides aminés indispensables qui pourraient être les plus limitants dans l'aliment Poisson $\mathrm{A}$, particulièrement de tryptophane et d'acides aminés ramifiés et aromatiques. L'aliment Soja est moins riche en énergie digestible (environ 5 p. IOO) que l'aliment Poisson A mais d'autres facteurs ont pu défavoriser son utilisation. Ainsi, plusieurs auteurs ont mis en évidence des anticorps spécifiques des protéines alimentaires dans le sang de veaux recevant les protéines de soja (VAN ADRICHEM et FRENS, I965; VAN LEEUWEN, WEIDE et BraAs, Ig69; Smith et Sissons, I975); Roy et al. (I977) ont obtenu un épaississement de la paroi de l'intestin grêle en remplaçant une partie des protéines du lait 
par celles du soja. Cependant, dans notre essai, aucun symptôme d'allergie n'a été observé chez les animaux du lot Soja.

En conclusion, les concentrats protéiques de poissons blancs préparés par hydrolyse enzymatique ménagée peuvent remplacer une part importante de la poudre de lait écrémé dans les aliments d'allaitement destinés au veau de boucherie. Cependant, pour éviter tout effet dépressif, il conviendrait probablement de ne pas dépasser un taux de substitution voisin de $35 \mathrm{p}$. Ioo après avoir associé ces concentrats à la poudre de lactosérum, afin d'obtenir un mélange mieux équilibré en acides aminés indispensables. Comme l'ont observé SEVE et al. (I978) chez le porcelet, une grande attention doit être portée aux conditions de l'hydrolyse, en particulier au choix de la préparation enzymatique, pour éviter d'obtenir des fractions peu utilisables par l'animal. Malgré sa composition en acides aminés plus favorable, le concentrat protéique de soja que nous avons étudié n'est pas aussi bien utilisé que le concentrat de poisson hydrolysé par les enzymes végétales; d'autres essais seraient nécessaires pour préciser son taux optimum d'incorporation.

Accepté pour publication en aồt 1979.

\section{Remerciements}

A. M. Mrché, I,aboratoire des Maladies Métaboliques, I.N.R.A. Theix, qui a effectué les dosages de glucose et d'urée plasmatiques.

\section{Summary}

The effect of the method of fish protein hydrolysis on their utilization in the veal calf Comparison with milk and soybean proteins.

The utilization of 3 protein concentrates (Fish A, Fish B and Soybean) (table I and 2) was studied in the veal calf. The fish concentrates were prepared from white fish by partial hydrolysis using a mixture of plant (A) or bacterial (B) enzymes. The soybean concentrate was a soybean oil-meal treated by moist heating and cleared of its sucrose and $\alpha$ galactosides by alcohol extraction.

Four milk replacers containing 24 p. roo crude protein and is p. Ioo fat on a dry matter basis, were prepared (table 2 and 3 ). In the control diet, the proteins were almost exclusively provided by skim milk. In the 3 other diets (Fish A, Fish B and Soybean) 59 P. I oo of the proteins were supplied by one of the protein concentrates studied, the rest by skim milk powder, lysine and methionine. Each diet was offered to I 2 Friesian bull calves from 3 to i 6 weeks of age.

The performances of the calves were lower with Fish A diet than with the control diet (table 4 and 6$)$, but only the difference in feed conversion ratio $(6.5$ p. 100 for the whole trial) was significant. The results obtained with Fish B diet were less satisfactory than those concerning Fish A diet $(-8,6 ;-4,9$ and $-5,1$ p. roo for liveweight gain, feed conversion ratio and carcass weight). The performances of the calves fed the soybean diet were very similar to those of the calves given Fish $\mathrm{B}$ diet and thus lower than those of the calves fed control and fish $\mathrm{A}$ diets. The haematocrit value decreased less with Fish $\mathrm{B}$ and soybean diets than with control and Fish A diets (fig. I).

The adverse effect of replacing milk protein by that of the protein concentrates studied might be partly due to an insufficient protein supply, at least during the first weeks of the trial, as indicated by the lower uraemia in the calves fed Fish A, Fish B and soybean diets (fig. I). It may also be explained by essential and semi-essential amino acid deficiencies, mainly in Fish A and Fish B diets, as suggested by measurements of blood levels of free amino-acids in the calves 
(table 5). The less good utilization of fish B comparatively to Fish A, might be due to a lower valine availability (table 5 ).

Protein concentrates prepared by enzymatic hydrolysis of white fish might replace large proportion of skim milk powder in milk replacers fed to veal calves. However, in order to avoid any adverse effect, the substitution level must not exceed $35 \mathrm{p}$. Ioo; it is also useful to mix these fish concentrates with whey powder so as to get a better essential amino acid composition. The kind of enzymes used for hydrolysis has an important effect on protein utilization. The soybean concentrate studied in this trial was not as well used as the Fish $\mathrm{A}$ concentrate.

\section{Références bibliographiques}

Adrichem (VAN) P. W. M., Frens A. M., I965. Soybean protein as alimentary antigen in fattening calves. Tijdschr. Diergeneesk., 90, 525-530.

Dodsworith T. L., OWen J. B., Mackie I. M., Richtie A., Orskov I. R., I977. Fish - protein hydrolysate as a substitute for milk protein in calf feeding. Anim. Prod., 25, I9-26.

FAICHNEY G. J., WEston R. H., I97I. Digestion by ruminant lambs of a diet containing formaldehyde treated casein. Austr. J. Agric. Res., 22, 46I-468.

GORRILL A. D. I., Nicholson J. W. G., I969. Growth, digestibility and nitrogen retention by calves fed milk replacers containing milk and soybean proteins, supplemented with methionine. Can. J. Anim. Sci., 49, 3I5-32I.

Grizard J., Prugnaud J., Arnal, M., Pion R., 1975. Effet de l'insuline sur la composition corporelle et les teneurs en acides aminés libres du sang, du foie et du muscle du rat en croissance soumis à une restriction énergétique. Ann. Biol. anim. Bioch. Biophys., 15, 569-582.

Guilioteau P., Paruelif J.-L., Touldeic R., Mathifu C.-M., 1975. Utilisation des protéines pat le veau préruminant à l'engrais. III. Influence du remplacement des protéines du lait par celles du poisson sur la vidange stomacale. Ann. Zootech., 24, 243-253.

Guilloteau P., TOUllec R., Culroli J., LE DOUARON D., I977. Utilisation des protéines pat le veau préruminant à l'engrais. V. Utilisation digestive des protéines du poisson, du soja et de la féverole. Ann. Zootech., 26, I7-3o.

Guilloteau P., Toullec R., SaUvant D., Paruelle J.-I., i979. Utilisation des protéines par le veau préruminant à l'engrais. VII. Influence $d u$ remplacement des protéines du lait par celles du soja ou de la féverole sur l'évacuation gastrique. Ann. Zootech., 28, r-17.

LEEUWEN (VAN) W. M., WEIDE H. J., BRAAS C. C., I969. Feeding value of soybean oil tneal compared with dried skim milk. Versl. Landbouw. Onderzock. Ned., 732, I-I8.

MicheL M., I973. Recherches de tests biochimiques destinés à caractériser l'état nutritionnel et sanitaire d'un troupeau de veaux. Ann. Rech. Vétér., 4, I I- 24.

Morrile J.-I., Meiton S. I., Dayton A. D., Guy E. J., Paliansch M. J., rg7i. Evaluation of milk replacers containing a soy protein concentrate and high whey. J. Dairy Sci., 54, I o6oI063.

Nitsan Z., Volcani R., Gordin S., Hasdai A., I97I. Growth and nutrients utilization by calves fed milk replacers containing milk or soybean protein concentrate heated to various degrees. J. Dairy Sci., 54, 1294-1299.

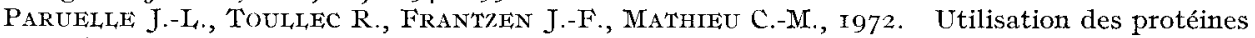
par le veau préruminant à l'engrais. I. Utilisation digestive des protéines du soja et des levures d'alcanes incorporées dans les aliments d'allaitement. Ann. Zootech., 21, 3I8-33I.

Paruelige J.-I., TOUl, Ec R., Patureau-Mirand P., I975. Utilisation des protéines par le veau préruminant à l'engrais. IV. Utilisation des protéines des levures d'alcanes et influence de l'addition d'un complexant du fer. Ann. Zootech., 24, 685-696.

Paruelife J.-L., Toullec R., Patureau-Mirand P., Mathinu C.-M., i974. Utilisation des protéines par le veau préruminant à l'engrais. II. Utilisation des protéines de poisson et influence de l'addition d'un complexant du fer. Ann. Zootech., 23, 519-536.

Patureau-Mirand P., Prugnaud J., Pion R., i97I. Influence de la nature des protéines des aliments d'allaitement sur l'aminoacidémie libre du veau préruminant. $X^{\mathrm{e}}$ Congrès Intern. Zootech., Thème I, Ruminants, Versailles.

Patureau-Mirand P., Toulifec R., Parueilife J.-L., Prugnaud J., Pion R., i974. Influence de la nature des matières azotées des aliments d'allaitement sur l'aminoacidémie du veau préruminant. I. Matières azotées du lait, du lactosérum, du poisson et des levures d'alcanes. Ann. Zootech., 23, 343-358.

PION R., DE BELSUNCE C., FACCONNEAU G., I963. Composition en acides aminés de quelques aliments. Ann. Biol. anim. Bioch. Biophys., 3 (h.s.), ir-1 8. 
Pion R., Fauconneau G., ig66. Les acides aminés des protéines alimentaires. Méthodes de dosage et résultats obtenus. In Amino acides, peptides, protéines. Cahier no 6, I59-I 75. A.E.C. Société de chimie organique et biologique, Commentry.

Roy J. H. B., Stobo I. J. F., Shotron S. M., Ganderton P., Girlies C. M., I977. The nutritive value of non-milk proteins for the preruminant calf. The effect of replacement of milk protein by soya-bean flour or fish-protein concentrate. Brit. J. Nutr., 38, I67-187.

Seve B., Aumaitre A., Jaubert P., Tord P., r978. Solubilisation des protéines de poisson, supplémentation en tryptophane et valeur alimentaire pour le porcelet. Ann. Zootech., 27, 423-437.

ShUMP P., Schreuder H. A. W., r969. Determination of tryptophan in foods. Analyt. Bioch., 27, I $82-186$.

SMITH R. H., Sissons J. W., 1975. Effects of different feeds, including those containing soyabean products, on the passage of digesta from the abomasum of the preruminant calf. Brit. J. Nutr., 33, 329-349.

TOUlLec R., Corollek J.-Y., Patureau-Mirand P., ig77. Utilisation des protéines par le veau préruminant à l'engrais. VI. Influence de la solubilité des protéines de maïs et de poisson sur leur utilisation digestive. Ann. Zootech., 26, 523-532.

TOUllec R., GUillotead P., COROLlér J.-Y., I979. Influence de la cinétique d'évacuation gastrique de l'aliment sur 1'absorption chez le veau préruminant. Ann. Biol. anim. Bioch. Biophys., 19, $729-732$.

Toullec R., PARUeile J.-L., COROLler J.-Y., LE TREu' J.-H., I977. Utilisation digestive par le veau préruminant de laits de remplacement contenant de 1'ultrafiltrat de lactosérum comme seule source de lactose. Ann. Zootech., 26, 29-43.

Troccon J.-L., Toul. EC R., I976. Influence du remplacement du laít écrémé par d'autres sources de protéines dans l'aliment d'allaitement distribué à des veaux femelles sevrés précocement. Bull. Techn. C.R.Z.V. Theix, I.N.R.A., 26, 55-6I.

WEERDEN (VAN) E. J., HuISMAN J., SPRIETSMA J. E., I973. De fysiologische beschikbaarheid van het ijzer in eiwitrijke groudstoffen bij meskalveren. Landbouw. Tijdsch., 85, 379-387.

WEERDEN (VAN) E. J., VAN ES A. J. H., VAN HELLEMOND K. K., I970. Eiwit-en vetaanzet van mestkalveren bij verschillende rantsoenen. Landbou». Tijdsch., 85, 379-387.

WILSON K. O., 1972. The nutritive value of enzymatic predigestion of fish protein concentrate for young calves. Thesis, Texas A. M. University, 89 pp. 